\title{
Rotational Velocities of S-Type Symbiotic Stars
}

\author{
Francis C. Fekel \\ Tennessee State University, Center of Excellence in Information \\ Systems, Nashville, Tennessee 37203, USA \\ Kenneth H. Hinkle and Richard R. Joyce \\ National Optical Astronomy Observatory, Tucson, Arizona 85726, USA
}

\begin{abstract}
We obtained high-resolution spectroscopic observations at $2.226 \mu \mathrm{m}$ of 13 S-type symbiotic binaries using the Phoenix infrared spectrograph on Kitt Peak telescopes. From an empirical calibration we determined $v \sin i$ values for the giant components. Combined with information from a program to determine orbits, the rotational velocities have been used to estimate the sizes of these stars and the fraction of their Roche lobes that they fill.
\end{abstract}

\section{Observations}

High-resolution spectroscopic observations of 13 symbiotic systems plus several standard stars having a range of $v \sin i$ values were obtained at $2.226 \mu \mathrm{m}$ with the Phoenix infrared spectrograph (Hinkle et al. 1998) on the 2.1 and $4 \mathrm{~m}$ Kitt Peak National Observatory telescopes. The wavelength region covers about $100 \AA$ and was chosen to contain several isolated atomic lines.

\section{Measurements and Inferences About Mass Exchange}

In each spectrum the full-width at half-maximum was measured for 3 or 4 isolated atomic features. The instrumental broadening was adopted from the width of several water vapor lines in the wavelength region. For the late-type giants with known $v \sin i$ values, we determined an empirical broadening relationship with a procedure similar to that of Fekel (1997). Using that empirical infrared calibration and an adopted macroturbulence of $3 \mathrm{~km} \mathrm{~s}^{-1}$, we obtained projected rotational velocities for the late-type giants in our observed symbiotic systems. Velocity uncertainties are estimated to be $1 \mathrm{~km} \mathrm{~s}^{-1}$ except for BF Cyg and V1329 Cyg, where the uncertainty is $2 \mathrm{~km} \mathrm{~s}^{-1}$.

Nearly all symbiotics with well-determined orbital elements have circular orbits. From the work of Zahn (1977) and following Schmutz et al. (1994), we assume that if the orbit of the symbiotic binary is circular or nearly so, the late-type giant in such a system is synchronously rotating and its rotational and orbital axes are aligned. With an estimate of the inclination taken from the literature the projected rotational velocity is converted to an equatorial rotational velocity. Then the radius $R$ of the late-type star can be determined 
from

$$
R=(v P) / 50.6
$$

where $R$ is in solar radii, $v$ is the rotational velocity in $\mathrm{km} \mathrm{s}^{-1}$, and $P$ is the orbital period in days. With orbital elements from our published (Fekel et al. 2000a,b; Fekel et al. 2001) and unpublished results plus masses taken from the literature or in some cases canonical values of 1.5 and $0.5 \mathrm{M}_{\odot}$ for the cool and hot components, respectively, the separation of the two stars follows from Kepler's third law. This leads to the computation of an effective Roche lobe radius from Eq. (2) of Eggleton (1983).

Table 1. Cool giant radius and Roche lobe radius comparison

\begin{tabular}{|c|c|c|c|c|c|c|c|c|}
\hline Star & $\begin{array}{r}\mathrm{P}_{\text {orb }} \\
\text { (days) }\end{array}$ & $\begin{array}{l}\mathrm{v} \sin \mathrm{i} \\
(\mathrm{km} / \mathrm{s})\end{array}$ & $\begin{array}{c}\mathrm{i} \\
\left(^{\circ}\right)\end{array}$ & $\begin{array}{c}\mathrm{R} \\
\left(\mathrm{R}_{\odot}\right)\end{array}$ & $\begin{array}{r}\mathrm{M}_{c} \\
\left(\mathrm{M}_{\odot}\right)\end{array}$ & $\begin{array}{r}\mathrm{M}_{h} \\
\left(\mathrm{M}_{\odot}\right)\end{array}$ & $\begin{array}{r}\mathrm{R}_{L} \\
\left(\mathrm{R}_{\odot}\right)\end{array}$ & $\mathrm{R} / \mathrm{R}_{L}$ \\
\hline TX CVn & 199 & 8.9 & 60 & 40 & 1.5 & 0.5 & 85 & 0.47 \\
\hline $\mathrm{T}$ CrB & 228 & 5.4 & 60 & 28 & 0.7 & 1.2 & 53 & 0.53 \\
\hline BF Cyg & 757 & 4.5 & 75 & 70 & 2.2 & 0.6 & 243 & 0.29 \\
\hline $\mathrm{CH}$ Cyg & 756 & 8.2 & 70 & 130 & 1.5: & 0.3 : & 221: & 0.59: \\
\hline CI Cyg & 854 & 10.4 & 80 & 180 & 1.5 & 0.5 & 227 & 0.79 \\
\hline V1329 Cyg & 956 & 7.0 & 86 & 132 & 2.2 & 0.75 & 277 & 0.48 \\
\hline AG Dra & 549 & 3.6 & 40 & 61 & 1.0 & 0.5 & 141 & 0.43 \\
\hline V443 Her & 599 & 4.5 & 60 & 62 & 1.5 & 0.5 & 179 & 0.35 \\
\hline RW Hya & 370 & 5.0 & 80 & 37 & 1.6 & 0.5 & 134 & 0.28 \\
\hline BX Mon & 1259 & 6.8 & 85 & $\ldots$ & $\ldots$ & $\ldots$ & $\ldots$ & $\ldots$ \\
\hline AG Peg & 818 & 4.5 & 60 & 84 & 2.6 & 0.65 & 273 & 0.31 \\
\hline FG Ser & 634 & 7.0 & 80 & 89 & 1.7 & 0.6 & 193 & 0.46 \\
\hline V343 Ser & 451 & 5.7 & 18 & 144: & 1.5 & 0.5 & 148 & 0.97: \\
\hline
\end{tabular}

Table 1 provides the results for the late-type giants in $13 \mathrm{~S}$-type symbiotic systems. The last column of the table gives the ratio of the giant's radius to its effective Roche lobe radius, indicating how much of the Roche lobe it fills. In agreement with the results of Mürset \& Schmid (1999), we find that most of the giants do not come close to filling their Roche lobes.

Acknowledgments. This research has been supported in part by NASA grant NCC5-511 and NSF grant HRD-9706268 to Tennessee State University.

\section{References}

Eggleton, P. P. 1983, ApJ 268, 368

Fekel, F. C. 1997, PASP 109, 514

Fekel, F. C., Hinkle, K. H., Joyce, R. R., \& Skrutskie, M. F. 2000a, AJ 120, 3255

Fekel, F. C., Joyce, R. R., Hinkle, K. H., \& Skrutskie, M. F. 2000b, AJ 119, 1375

Fekel, F. C., Hinkle, K. H., Joyce, R. R., \& Skrutskie, M. F. 2001, AJ 121, 2219

Hinkle et al. 1998, SPIE 3354, 810

Mürset, U., \& Schmid, H. M. 1999, A\&AS 137, 473

Schmutz, W., Schild, H., Mürset, U., \& Schmid, H. M. 1994, A\&A 288, 819

Zahn, J. P. 1977, A\&A 57, 383 